\title{
Mesonephric carcinoma of the uterine corpus: A report of two cases
}

\author{
JIANGUO ZHAO ${ }^{1,2}$, CAIYAN LIU ${ }^{2}$, JI QI ${ }^{2}$ and PENGPENG QU ${ }^{2}$ \\ ${ }^{1}$ Clinical College of Central Gynecology and Obstetrics, Tianjin Medical University, Heping, Tianjin 300070; \\ ${ }^{2}$ Department of Gynecological Oncology, Tianjin Central Hospital of Gynecology and Obstetrics, Tianjin 300100, P.R. China
}

Received October 16, 2014; Accepted August 17, 2015

DOI: 10.3892/ol.2015.3886

\begin{abstract}
Mesonephric carcinoma of the uterine corpus is an extremely rare malignancy that originates from the mesonephric remnant of the female reproductive tract. There have been no previous reports of mesonephric carcinoma accompanied by a sarcomatous component. The current study presents two cases of uterine corpus mesonephric carcinoma with sarcomatous components that occurred in postmenopausal women. Immunohistochemically, the tumour cells were positive for vimentin and CD10. The tumours of these patients were located in the uterine myometrium; therefore, treatment was similar to that for endometrial cancer. Although the follow-up period was short in the current cases, no metastatic disease was identified in the second case and thus, this may increase clinical knowledge with regard to the biological behavior of these rare malignancies.
\end{abstract}

\section{Introduction}

Mesonephric carcinoma of the uterine corpus is a rare malignancy that originates from the mesonephric remnant of the female reproductive tract (1). The mesonephric duct, which parallels the paramesonephric duct during the embryonic stage, forms the male epididymis, vas deferens, seminal vesicles, partial prostate and urethra (2). Due to the lack of androgens, the mesonephric duct degenerates in female embryos. The mesonephric remnant in females is typically located in the ovary, broad ligament and cervical sidewalls, although it may rarely be located in the vaginal wall or myometrium (2). Due to the limited number of cases reported of mesonephric carcinoma occurring within the myometrium, there is limited information regarding the diagnosis and

Correspondence to: Mrs. Pengpeng Qu, Department of Gynecological Oncology, Tianjin Central Hospital of Gynecology and Obstetrics, 156 Nankai Third Road, Nankai, Tianjin 300100, P.R. China

E-mail: qu.pengpeng@hotmail.com

Key words: mesonephric carcinoma, uterine corpus, sarcomatous component, diagnosis, treatment treatment of this malignancy. In the present report, 2 cases of mesonephric carcinoma diagnosed in the Tianjin Central Hospital of Gynecology and Obstetrics (Tianjin, China) are presented.

\section{Case report}

Case 1. A 55-year-old female was admitted to the Tianjin Central Hospital of Gynecology and Obstetrics (Tianjin, China) in October 2013 for postmenopausal bleeding. The dilation and curettage pathology report from another hospital revealed poorly-differentiated endometrial adenocarcinoma with mucinous degeneration and local mesenchymal dysplasia with sarcomatoid carcinoma in the uterine cavity. Histological analysis of the uterus by a pathologist at the Tianjin Central Hospital of Gynecology and Obstetrics indicated a diagnosis of mesonephric carcinoma. The patient had been diagnosed with diabetes mellitus 5 years earlier, according to the medical history. The physical examination of the patient was normal, and the bimanual vaginal examination indicated that the vulva, vagina and cervix were normal, and the uterus was anteverted, large, mobile, bilateral, and free of parametrial involvement. Pelvic ultrasonography revealed a vesicle in the left ovary and a hypoechoic area in the posterior uterine wall, although blood flow was not observed. Pelvic magnetic resonance imaging revealed the presence of an abnormally enhanced signal in the uterine cavity, hypervascular lesions in the uterine corpus and isthmus and free fluid in the pelvis. Regarding the analysis of tumour markers, the levels of carbohydrate antigen (CA) 125, CA19-9 and $\alpha$-fetoprotein (AFP) were $163.8 \mathrm{U} / \mathrm{ml}$ (normal, 0-35 U/ml), $193.6 \mathrm{U} / \mathrm{ml}$ (normal, 0-27 U/ml) and $16.8 \mathrm{ng} / \mathrm{ml}$ (normal, 0-7 ng/ml), respectively. In consequence, extra-fascia hysterectomy, bilateral salpingo-oophorectomy and pelvic lymphadenectomy were performed. The intraoperative findings revealed ascites $(\sim 300 \mathrm{ml})$ and a large uterus. The bilateral ovaries and oviducts, pelvic lymph nodes, liver, spleen and diaphragmatic surface were all normal. A nodular mass of $3.5 \times 2.0 \times 2.5 \mathrm{~cm}$ in size was observed in the lower one-third of the uterus and the cervix. The mass invaded the myometrium, and the deepest part of the invasion almost reached the serous layer. The microscopic analysis of the tumour resulted in a diagnosis of mesonephric carcinoma with a sarcomatoid component, which involved the cervical stroma and $>50 \%$ of the uterine myometrium near the serosa. No involvement 

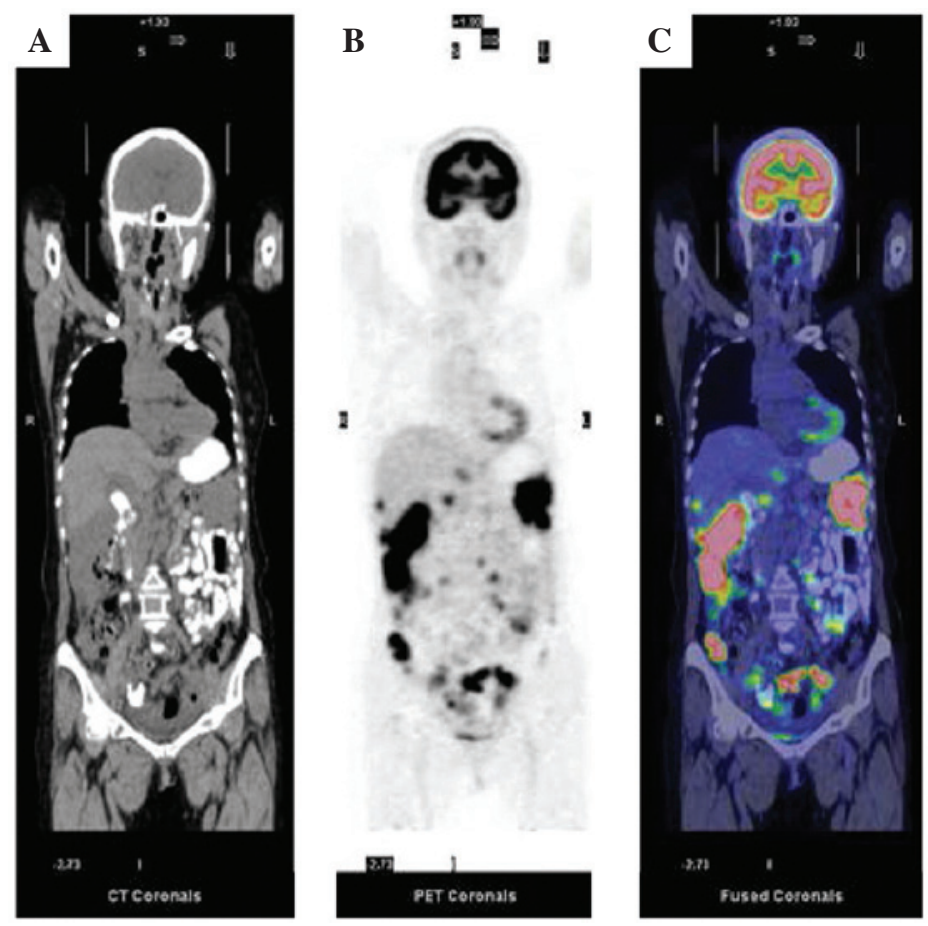

D

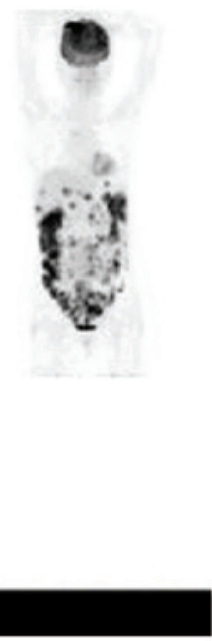

Figure 1. Case 1: Whole body 18F-flutemetamol contrast-enhanced PET/CT imaging following surgery. (A) Coronal CT showing multiple lesions, (B) coronal PET imaging showing abnormal radioactive accumulation and (C) coronal combined PET and CT imaging showing enhanced 18F-flutemetamol uptake, in the abdomen and pelvis, including the liver capsule, lesser omentum, splenic capsule, peritoneummesentery, right paracolic sulci, the pouch of Douglas and the pelvic peritoneum. (D) Maximum intensity projection of PET/CT imaging showing metastatic lesions (black areas) in the abdominal and pelvic cavity. PET, positron emission tomography; CT, computed tomography.

of the bilateral ovaries and oviducts, lymphovascular space, bilateral parametrium or pelvic lymph nodes, was observed. On immunohistochemical analysis, the tumour specimen was positive for cluster of differentiation (CD) 10, calretinin, vimentin, cytokeratin $(\mathrm{CK})$ and epithelial membrane antigen; and negative for estrogen receptor (ER), progesterone receptor (PR), CD99, and Wilms tumour-1. Furthermore, the ascites cytology was positive, showing the presence of tumour cells. Therefore, the postoperative diagnosis was mesonephric carcinoma. Chemotherapy, consisting of paclitaxel $(270 \mathrm{mg})$ on day 1 , and cisplatin $(20 \mathrm{mg})$ and ifosfamide $(2 \mathrm{~g})$ on days $2-5$, was subsequently administered to the patient. After the first cycle of chemotherapy, the patient's CA125 and CA19-9 levels had normalized to $31.02 \mathrm{U} / \mathrm{ml}$ and $25.24 \mathrm{U} / \mathrm{ml}$, respectively. The second cycle of chemotherapy, comprising paclitaxel and cisplatin, was modified due to severe myelosuppression, resulting in the administration of 4 cycles of paclitaxel (270 mg; day 1) and cisplatin (50 mg; days 2-3) chemotherapy. Subsequent to treatment, the patient received regular follow-up examinations. At 8 months post surgery, a pelvic ultrasonogram revealed a hypoechoic mass in the pelvis of 58x36x33 mm in size. The patient's CA125 and CA19-9 levels had risen to $305.90 \mathrm{U} / \mathrm{ml}$ and $60.78 \mathrm{U} / \mathrm{ml}$, respectively. Positron emission tomography (PET)-computed tomography performed at the Tianjin Medical University Cancer Institute and Hospital (Tianjin, China) in April 2014 revealed possible metastasis to the mediastinal lymph nodes, liver capsule, splenic capsule, liver falciform ligament, hepatogastric ligament, lesser omentum, peritoneum, mesentery and Douglas fossa (Fig. 1). Therefore, a re-challenge chemotherapy regimen of pirarubicin (50 mg; day 1), cisplatin (20 mg; days 2-5) and ifosfamide (2 g; days 2-5) was subsequently administered. The patient succumbed to the disease in April 2014, after an overall survival time of almost 12 months.

Case 2. A 62-year-old female was admitted to the Tianjin Central Hospital of Gynecology and Obstetrics for postmenopausal bleeding in April 2014. Based on the dilation and curettage pathology report, a diagnosis of mesonephric carcinoma was considered. The patient presented with a 20 -year history of hypertension. The physical examination was normal, and the bimanual vaginal examination revealed that the vulva, vagina and cervix were normal, and the uterus was anteverted, large, mobile, bilateral, and free of parametrial involvement. An intrauterine mass of $58 \times 38 \times 37 \mathrm{~mm}$ in size was observed by pelvic ultrasonography, and submucosal uterine fibroids were considered. Pelvic magnetic resonance imaging revealed abnormal signal enhancement in the uterine cavity, which was indicative of malignancy. In addition, multiple small lymph nodes were observed around the iliac vessels and in the groin. The levels of carcinoembryonic antigen were $10.52 \mathrm{ng} / \mathrm{ml}$ (normal, 0-7 ng/ml), while the levels of other tumour markers were normal (CA125, 31.02 U/ml; CA19-9, $25.24 \mathrm{U} / \mathrm{ml}$; AFP, $3.7 \mathrm{ng} / \mathrm{ml}$ ). In consequence, extra-fascia hysterectomy, bilateral salpingo-oophorectomy and pelvic lymphadenectomy were performed. The intraoperative findings revealed mild bloody ascites and a large uterus. The bilateral ovaries and oviducts, pelvic lymph nodes, liver, spleen and diaphragmatic surface were all normal. The pathological analysis resulted in a diagnosis of mesonephric carcinoma of the uterine corpus with sarcomatoid components and no lymphovascular involvement. On immunohistochemical analysis, the tumour specimen was 
positive for CK, CK8/18, CD10, P16 and vimentin, and negative for ER, PR, CD15, P53 and calretinin. In addition, the tumour was partially positive for Ki-67 ( $<5 \%$ in the epithelial area and $60 \%$ in the spindle cell sarcoma area). The tumour involved the left internal iliac lymph node, but not the bilateral ovaries, oviducts or parametrium. Tumour cells were not detected in the ascites. Postoperatively, CEA levels returned to normal values. The patient subsequently received chemoradiation therapy with docetaxel (100 mg; day 1) and cisplatin (50 mg; days 2-3). The patient has shown no evidence of disease to date.

\section{Discussion}

Mesonephric carcinoma is a rare malignancy of the female genital tract. Compared with mesonephric carcinoma of the uterine corpus, cervical mesonephric carcinoma is more frequently observed, with 27 of the 29 cases of mesonephric carcinoma reported in the English literature occurring in the cervix, and 2 in the corpus (2). In 2013, Meguro et al (3) summarized 42 cases of cervical mesonephric adenocarcinoma, including 10 cases with sarcomatous components and 4 cases with exogenous or polypoid tumours. At present, only 3 cases of uterine corpus mesonephric carcinoma have been reported in the literature (Table I) $(1,2,4)$. Furthermore, to the best of our knowledge, no cases of mesonephric carcinoma accompanied by sarcoma components have been reported thus far.

In the 3 previous cases of mesonephric carcinoma of the uterine corpus, the ages of the patients at the time of diagnosis were 33,73 and 81 years, respectively $(1,2,4)$. The symptoms at presentation included abnormal vaginal bleeding, lower abdominal pain and presence of an abdominal mass. As mesonephric carcinoma of the uterine corpus originates from the mesonephric remnant in the myometrium, growth of the tumour into the uterine cavity may cause abnormal vaginal bleeding, as observed in these patients. A pelvic ultrasound may reveal deformation of the uterus, presence of an intrauterine mass or other pelvic abnormalities. However, in order to obtain a definitive pathological diagnosis, tumour tissue must be obtained through dilation and curettage or hysteroscopy. If the tumour grows into the myometrium, an abdominal mass, abdominal pain, menorrhagia and other symptoms may develop. Thus, it is not easy to differentiate mesonephric carcinoma from adenomyosis; although a pelvic ultrasound may reveal an enlarged uterus, the morphology of the uterine cavity may be normal, and a hysteroscopic examination may also be normal; and if the tumour tissue is lost then a pathological diagnosis cannot be made. Mesonephric carcinoma of the uterine corpus has been previously reported not to exhibit any ultrasonographic manifestation. However, ultrasonographic indications of mesonephric carcinoma in the appendages have been previously reported in the literature, including a mixed mass in the annex area (5). In addition, blood vessels may or may not be displayed on Doppler imaging (5). The 2 patients of the present case report, who were aged 55 and 62 years old, respectively, presented with the same symptom of postmenopausal bleeding. In the first patient, ultrasonography indicated possible uterine fibroids; however, no abnormal mass appeared within the uterine cavity, and the uterine fibroids presented without vessel a signal. In the second patient, ultrasonography suggested possible submucosal fibroids. These findings indicate that there

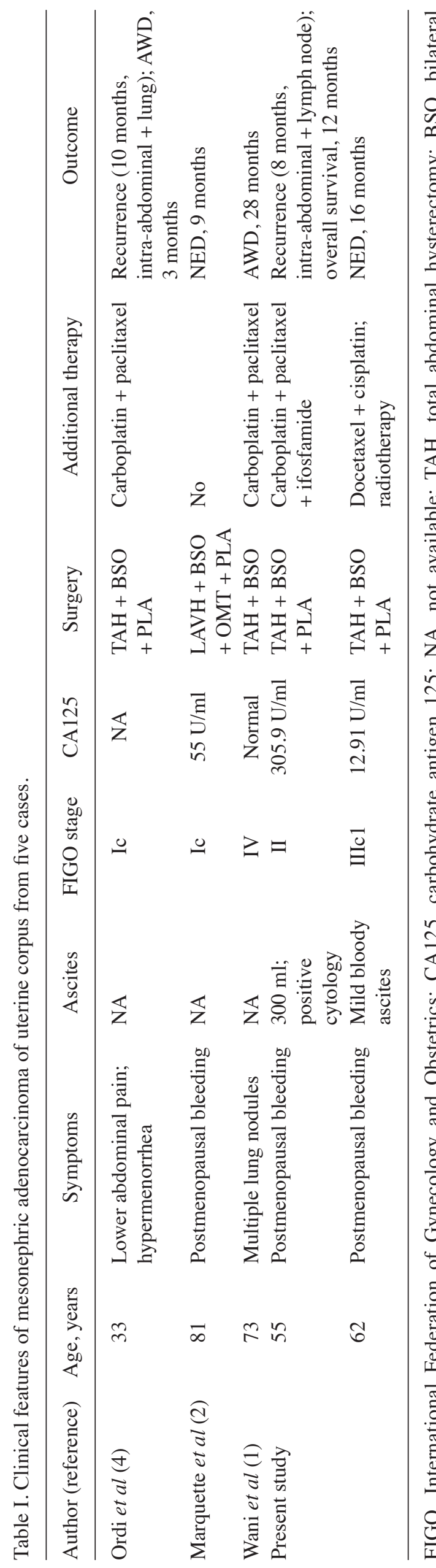

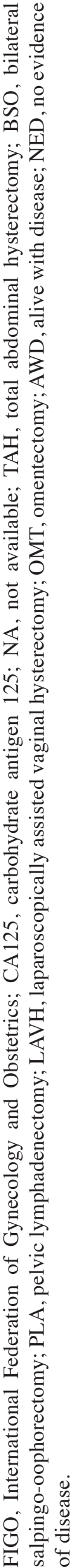


are certain limitations to the diagnosis of mesonephric carcinoma by ultrasonography alone.

The serum levels of CA125 have been reported to be slightly increased in cases of mesonephric carcinoma of the uterine corpus. However, serum CA125 is not a specific indicator of this malignancy (2), as normal levels of CA125 were observed in a previous case of mesonephric carcinoma of the uterine corpus presenting with lung metastases (1). In the present case report, the levels of CA125 were $163.8 \mathrm{U} / \mathrm{ml}$ in the first patient, and normal in the second patient, indicating that serum CA125 is not a specific marker for the diagnosis of corpus mesonephric carcinoma. Since mesonephric carcinoma originates from the mesonephric remnant present during the embryonic period, which is homologous with differential tissue from the endoderm (1), we hypothesize that the levels of other tumour markers such as AFP and carcinoembryonic antigen may also be elevated in mesonephric carcinoma, due to homology. The patient of the first case described in the present report experienced disease recurrence, as indicated by her elevated levels of CA125. Therefore, monitoring the levels of CA125 may be beneficial for assessing possible recurrence in patients with mesonephric carcinoma.

Due to the limited number of cases of uterine corpus mesonephric carcinoma reported to date, no standard surgical guidelines to treat this condition have been established thus far. Since the tumours of the 2 patients of the present case report were located in the uterine myometrium, the patients were accordingly subjected to the same type of surgery that is usually performed to treat endometrial cancer. This procedure differs from omentectomy, which has been previously reported to be conducted on a patient with corpus mesonephric carcinoma (2). Omentectomy remains a controversial procedure for the treatment of corpus mesonephric carcinoma; however, there has been one case report of a patient undergoing this procedure (2). Although it is considered that the omentum and the mesonephric remnant are homologous in terms of embryology (2), if the tumor does not invade the serous layer or if there is no omental involvement, then there is no mesonephric remnant located in the omentum $(1,4)$. Therefore, removal of the omentum may not be required. In the first patient of the present case report, the peritoneal ascites was positive for malignancy, and the patient experienced postoperative recurrence. Therefore, we believe that omentectomy should be performed in those patients with positive peritoneal lavage.

On microscopic examination of the tumour tissue, a small, tubular, mesh, solid, sex cord-like structure may be observed in cases of mesonephric carcinoma $(1,2,4)$. In addition, various cellular morphologies may also coexist within the same tumour (1). In particular, a tubular luminal epithelium and glandular cavity filled with eosinophilic material may be observed. Previous immunohistochemical analyses have revealed that tumour cells derived from mesonephric carcinoma are generally positive for vimentin, calretinin, inhibin and CD10, and tend to be negative for epithelial membrane antigen, ER, PR and CK20 (1,4). In the first case of the present report, the tumour specimen was positive for CD10, calretinin and vimentin, and negative for ER and PR; whereas the tumour specimen for the second case was positive for CD10 and vimentin, and negative for calretinin, ER and PR. These results were similar to those previously reported in the literature (4).
The most effective adjuvant chemotherapeutic regimen for the treatment of mesonephric carcinoma remains unclear. However, in previous reports, patients have been treated with regimens that included paclitaxel and carboplatin $(1,6)$. Among the cases of mesonephric carcinoma previously reported in the literature, 1 patient experienced local recurrence in the pelvis and metastasis to the lung 10 months post-surgery; 1 patient who presented lung metastases prior to surgery survived 28 months with the disease; and 1 patient demonstrated no evidence of recurrence within 9 months of follow-up (1,2). In another report (3), a patient with cervical mesonephric carcinoma with a sarcomatous component was treated with radical hysterectomy, bilateral salpingo-oophorectomy and pelvic lymph node dissection. The patient did not receive postoperative adjuvant chemotherapy, and presented with disease recurrence on the vaginal vault 7 months later. Subsequently, radiotherapy and chemotherapy were concurrently administered to the patient, and the recurrent tumour disappeared 3 months later. Montagut et al (6) reported a case of corpus mesonephric carcinoma treated with radical hysterectomy, bilateral salpingo-oophorectomy, para-aortic lymph node dissection and local postoperative radiotherapy, who developed pelvic-abdominal recurrence and lung metastasis 10 months later. Following 3 cycles of paclitaxel and carboplatin and surgical removal of the lesion, the patient achieved partial remission, and complete remission was achieved following 3 additional courses of chemotherapy. The patient in the first case of the present report experienced relapse 8 months post surgery, similar to the outcomes of other patients with mesonephric carcinoma previously reported in the literature (6); however, her overall survival time was almost 12 months post surgery. The second patient of the present case report, who presented with positive pelvic lymph nodes, demonstrated no evidence of recurrence within 16 months of follow-up.

In conclusion, female genital mesonephric carcinoma is a rare malignant tumour derived from embryonic mesonephric remnants. According to the literature, mesonephric carcinoma occurs more commonly in the cervix than in the uterus and vagina. The clinical symptoms associated with mesonephric carcinoma are not specific. In addition, imaging is unable to differentiate between this malignancy and common female reproductive system tumours, and no specific tumour markers for mesonephric carcinoma have been identified thus far. Therefore, the surgical treatment and staging of this malignancy may require to be based on the existent guidelines for endometrial cancer. Furthermore, patients with mesonephric carcinoma who exhibit risk factors should receive appropriate postoperative treatments.

\section{Acknowledgements}

Theauthors wish to thankEditage(http://online.editage.cn/) for English language editing, and the Department of Molecular Imaging and Nuclear Medicine, Tianjin Medical University Cancer Institute and Hospital, for providing PET images.

\section{References}

1. Wani Y, Notohara K and Tsukayama C: Mesonephric adenocarcinoma of the uterine corpus: A case report and review of the literature. Int J Gynecol Pathol 27: 346-352, 2008. 
2. Marquette A, Moerman P, Vergote I and Amant F: Second case of uterine mesonephric adenocarcinoma. Int J Gynecol Cancer 16: 1450-1454, 2006.

3. Meguro S, Yasuda M, Shimizu M, Kurosaki A and Fujiwara K Mesonephric adenocarcinoma with a sarcomatous component a notable subtype of cervical carcinosarcoma: A case report and review of the literature. Diagn Pathol 8: 74-78, 2013.

4. Ordi J, Nogales FF, Palacin A, Márquez M, Pahisa J, Vanrell JA and Cardesa A: Mesonephric adenocarcinoma of the uterine corpus: CD10 expression as evidence of mesonephric differentiation. Am J Surg Pathol 25: 1540-1545, 2001.
5. Kuper SG, Wright DR, Callahan MJ and Hiett AK: Ultrasound presentation of a female adnexal tumor of probable wolffian origin (FATWO). J Diagn Med Sonogr 29: 282-284, 2013.

6. Montagut C, Mármol M, Rey V, Ordi J, Pahissa J, Rovirosa A Gascón P and Mellado B: Activity of chemotherapy with carboplatin plus paclitaxel in a recurrent mesonephric adenocarcinoma of the uterine corpus. Gynecol Oncol 90: 458-461, 2003. 